University of Wollongong

Research Online

Australian Institute for Innovative Materials -

Papers

Australian Institute for Innovative Materials

$1-1-2016$

Rectification magnetoresistance device: Experimental realization and theoretical simulation

\author{
Kun Zhang \\ Shandong University \\ Qikun Huang \\ Shandong University \\ Yi Yan \\ University of California, Santa Barbara, yy619@uowmail.edu.au \\ Xiaolin Wang \\ University of Wollongong, xiaolin@uow.edu.au \\ Jing Wang \\ Shandong University
}

See next page for additional authors

Follow this and additional works at: https://ro.uow.edu.au/aiimpapers

Part of the Engineering Commons, and the Physical Sciences and Mathematics Commons

Research Online is the open access institutional repository for the University of Wollongong. For further information contact the UOW Library: research-pubs@uow.edu.au 


\title{
Rectification magnetoresistance device: Experimental realization and theoretical simulation
}

\author{
Abstract \\ A unique technique has been proposed to realize rectification magnetoresistance (RMR) by combining a \\ commercial diode and a magnetoresistance component in parallel. The observed RMR could be greatly \\ tuned in a wide range by applying direct current and alternating current simultaneously to the device. \\ Moreover, a quantitative theoretical model has been established, which well explained both the observed \\ RMR and the electrical manipulation behavior. The highly tunable RMR and the correlated magnetoelectric \\ functionalities provide an alternative route for developing multi-functional spintronics devices. \\ Disciplines \\ Engineering | Physical Sciences and Mathematics

\section{Publication Details} \\ Zhang, K., Huang, Q., Yan, Y., Wang, X., wang, J., Kang, S. \& Tian, Y. (2016). Rectification \\ magnetoresistance device: Experimental realization and theoretical simulation. Applied Physics Letters, \\ 109 (21), 213503-1-213503-5.
}

\section{Authors}

Kun Zhang, Qikun Huang, Yi Yan, Xiaolin Wang, Jing Wang, Shi-Shou Kang, and Yu-Feng Tian 


\section{Rectification magnetoresistance device: Experimental realization and theoretical simulation}

Kun Zhang, Qikun Huang, Yi Yan, Xiaolin Wang, Jing Wang, Shishou Kang, and Yufeng Tian

Citation: Appl. Phys. Lett. 109, 213503 (2016); doi: 10.1063/1.4968784

View online: http://dx.doi.org/10.1063/1.4968784

View Table of Contents: http://aip.scitation.org/toc/apl/109/21

Published by the American Institute of Physics

\section{Articles you may be interested in}

Spin-orbit torques in perpendicularly magnetized Ir22Mn78/Co20Fe60B20/MgO multilayer Appl. Phys. Lett. 109, 222401222401 (2016); 10.1063/1.4968785






\title{
Rectification magnetoresistance device: Experimental realization and theoretical simulation
}

\author{
Kun Zhang, ${ }^{1}$ Qikun Huang, ${ }^{1}$ Yi Yan, ${ }^{2}$ Xiaolin Wang, ${ }^{3}$ Jing Wang, ${ }^{1}$ Shishou Kang, ${ }^{1}$ \\ and Yufeng Tian ${ }^{1, a)}$ \\ ${ }^{1}$ School of Physics, State Key Laboratory of Crystal Materials, Shandong University, Jinan 250100, \\ People's Republic of China \\ ${ }^{2}$ Electrical and Computer Engineering, Harold Frank Hall, Room 4155, University of California, \\ Santa Barbara, California 93106-9560, USA \\ ${ }^{3}$ Institute of Superconducting and Electrical Materials, Australian Institute of Innovative Materials, \\ University of Wollongong, Wollongong, NSW 2522, Australia
}

(Received 11 September 2016; accepted 9 November 2016; published online 22 November 2016)

\begin{abstract}
A unique technique has been proposed to realize rectification magnetoresistance (RMR) by combining a commercial diode and a magnetoresistance component in parallel. The observed RMR could be greatly tuned in a wide range by applying direct current and alternating current simultaneously to the device. Moreover, a quantitative theoretical model has been established, which well explained both the observed RMR and the electrical manipulation behavior. The highly tunable RMR and the correlated magnetoelectric functionalities provide an alternative route for developing multi-functional spintronics devices. Published by AIP Publishing.

[http://dx.doi.org/10.1063/1.4968784]
\end{abstract}

In order to satisfy the ever-increasing demand for high data processing speed, low power consumption, and high integration densities in information storage and processing technology, various spintronics devices have been widely explored. Among them, the multi-functional devices with fascinating magnetoelectric coupling have attracted increasing attention for their ability to realize electrical manipulation of magnetism and magneto-transport properties. For example, as a result of the spin transfer torque effect, spin polarization current has been demonstrated as an efficient method to reverse the magnetic moment ${ }^{1-3}$ and to drive domain wall motion, ${ }^{4}$ which has promising applications in magnetic random access memories (MRAMs) and racetrack memories. ${ }^{5}$ In the meantime, multiferroic composites that exhibit more than one ferroic order have been widely investigated because of their intricate magnetoelectric coupling or magnetostrictive coupling. ${ }^{6-10}$ In fact, exciting progress has been achieved, where electrical manipulation of the saturation magnetization, Curie temperature, magnetic anisotropy, and exchange bias has been demonstrated as a result of the electrical field dependent charge accumulation, orbital reconstruction, and/or interfacial exchange coupling. ${ }^{11-16}$ Furthermore, voltage driven reversible ion migration could lead to promising non-volatile controlling of magnetism and resistance. $^{17-20}$

Recently, a promising rectification magnetoresistance (RMR) was reported in nonmagnetic $\mathrm{Al} / \mathrm{Ge}$ Schottky heterojunctions, ${ }^{21}$ i.e., the application of a pure alternating current (AC) can generate a significant direct current (DC) voltage which varies with the external magnetic field. The discovery of the RMR effect not only adds a new-member to the longhistorical magnetoresistance (MR) family but also provides an alternative approach to realize functional spintronics

\footnotetext{
a) Author to whom correspondence should be addressed. Electronic Mail: yftian@sdu.edu.cn
}

devices by using alternating current. In principle, RMR requires that the rectification effect and MR effect coexist in one device, so it is expected to exist in p-n junctions, n-n junctions, and Schottky diodes with large MR effect ${ }^{22,23}$ and magnetic tunnel junctions with the asymmetric barrier. ${ }^{24}$ Although the rectifying property and remarkable positive MR have been observed separately in $\mathrm{La}_{0.33} \mathrm{Ca}_{0.67} \mathrm{MnO}_{3} /$ $\mathrm{NbSrTiO}_{3}$ heterojunctions, ${ }^{23}$ the possible larger rectification magnetoresistance was not studied. Moreover, the remarkable MR is only found at low temperature and under certain bias voltage in these $n-n$ junctions, which limits their practical application. For realistic applications, room temperature operation, multi-degree of freedom control, and compatibility with current semiconductor technology are preferred.

Here, we proposed a unique technique to realize RMR by parallel connection of a commercial diode and a magnetoresistance component, both of which are fundamental electric elements in modern electronic industry. Furthermore, it is found that the room temperature RMR of the fabricated device is greatly tuned from $-11300 \%$ to $13500 \%$ by applying DC and AC simultaneously. A quantitative theoretical model was established by using physical parameters of the individual diode and MR component, which well explained the experimentally observed RMR. The state-ofthe-art technique could be used to develop multi-functional spintronic devices by using conventional fundamental electric elements.

In our experiments, we used $\mathrm{Zn}_{0.3} \mathrm{Co}_{0.7} \mathrm{O}$ amorphous magnetic semiconductor $(\mathrm{ZnCoO})$ films as an example of the magnetoresistance component, which were prepared on glass substrates by an alternating sputtering technique. ${ }^{25}$ The $\mathrm{ZnCoO}$ film of $60 \mathrm{~nm}$ in thickness was cut into a stripe with $4 \mathrm{~mm}$ in length and $2 \mathrm{~mm}$ in width. The rectification component is a commercial Schottky diode (1N5817) with a typical junction capacitance of $110 \mathrm{pF}$. The RMR devices were fabricated by combining the Schottky diode and $\mathrm{ZnCoO}$ film in 
parallel. The RMR and electrical manipulation of RMR were measured by using the Keithley 6221 AC current source to provide a sinusoidal AC input with/without DC offset and a Keithley 2182A voltmeter to detect the generated DC voltage. The current versus voltage (I-V) curves and conventional MR were measured with a Keithley 2400 DC current source and a Keithley 2182A voltmeter. All the transport properties were measured by using the two-point method with the external magnetic field applied in the film plane and parallel to the current direction.

Figure 1(a) shows the room temperature I-V curves of the $\mathrm{ZnCoO}$ film measured under different magnetic fields. Linear I-V curves were observed and the slope of I-V curves increases slightly with increasing magnetic field, revealing the absence of rectification effect and the existence of the MR effect. As shown in Fig. 1(b), a negative conventional MR $(-5 \%)$ originated from the spin dependent hopping ${ }^{25}$ was observed, and the rectification voltage was zero for $\mathrm{AC}=10 \mu \mathrm{A}$. This unambiguously indicates that only the MR


(c)

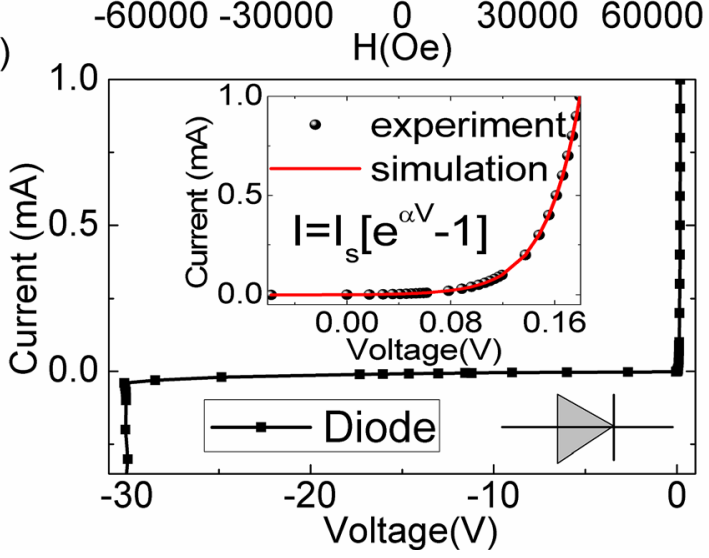

FIG. 1. (a) The experimental and theoretical fitting I-V curves of the $\mathrm{ZnCoO}$ film. (b) The conventional MR measured under $\mathrm{DC}=10 \mu \mathrm{A}$ and magnetic field dependent rectification voltage of the $\mathrm{ZnCoO}$ film measured under $\mathrm{AC}=10 \mu \mathrm{A}$. (c) The experimental $\mathrm{I}-\mathrm{V}$ curve of the commercial diode. The inset in (c) shows the theoretical fitting to the I-V curve of the commercial diode. effect without the rectification effect cannot induce any RMR. Here, MR is always defined as MR $=\frac{V_{H}-V_{0}}{V_{H}} \times 100 \%$, where $V_{H}$ and $V_{0}$ are the DC voltage detected with and without the applied magnetic field, respectively. In Fig. 1(c), the transport properties of the commercial diode have been characterized, where obvious rectification behavior with a forward opening voltage of $0.1 \mathrm{~V}$ and a reverse breakdown voltage of $30 \mathrm{~V}$ is observed.

In order to combine the rectification and MR effect, the $\mathrm{ZnCoO}$ film and commercial diode were connected in parallel to form a RMR device as schematically shown in the inset of Fig. 2(a). The non-linear I-V curves of the studied RMR device are shown in Fig. 2(a). Significant asymmetry for the positive and negative branch of $\mathrm{I}-\mathrm{V}$ curves indicates the existence of rectifying behavior. Meanwhile, the voltage measured at $6 \mathrm{~T}$ magnetic field is smaller than that measured without magnetic field, revealing the existence of the MR effect. Fig. 2(b) further shows the conventional DC MR measured under $\mathrm{DC}= \pm 10 \mu \mathrm{A}$. It is obvious that both the absolute value of the detected voltage and the MR ratio are different from each other, which is consistent with the asymmetrical I-V curves. Fig. 2(c) shows one of the most intriguing parts of current investigation, i.e., a $-13.3 \%$ RMR was
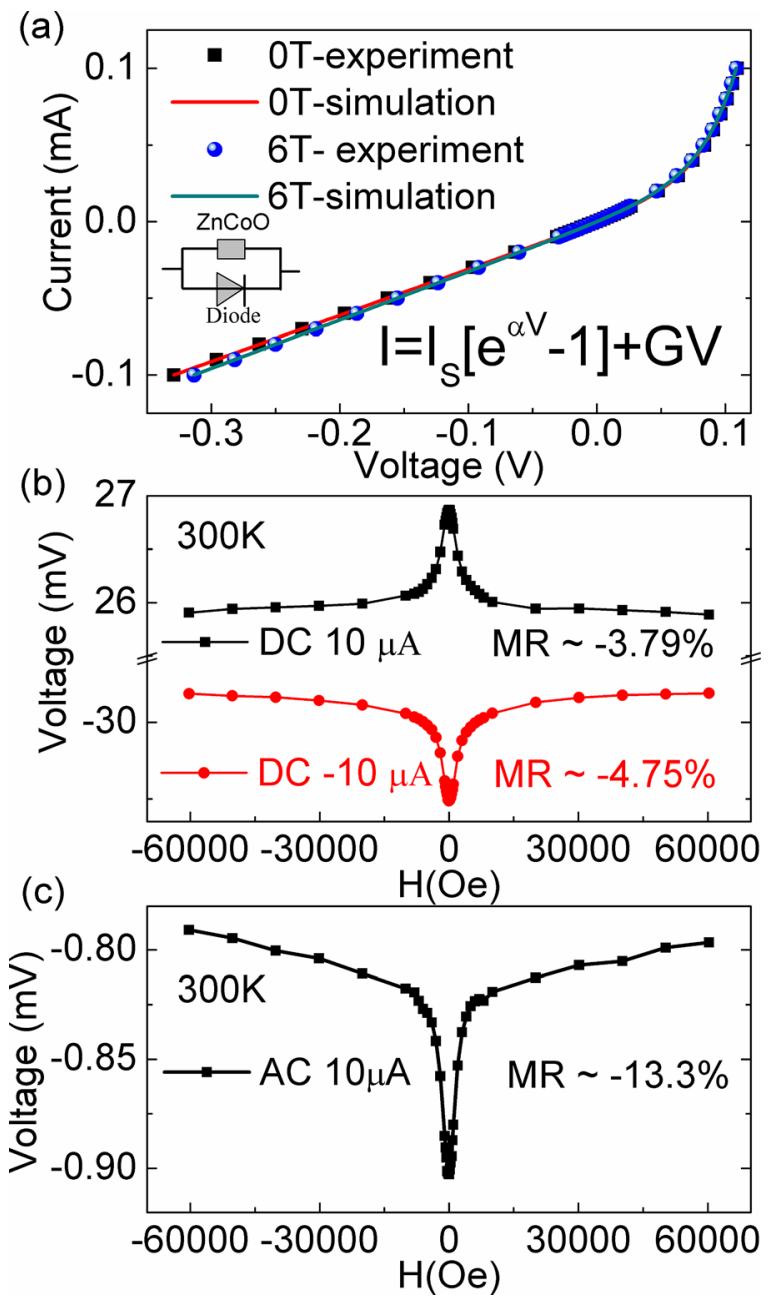

FIG. 2. (a) The experimental and theoretical simulated I-V curves of the RMR device. (b) The conventional MR of the RMR device. (c) The magnetic field dependent rectification voltage of the RMR device measured under fixed $\mathrm{AC}$ current $=10 \mu \mathrm{A}$. 
observed under $\mathrm{AC}=10 \mu \mathrm{A}$, which is a larger change than the conventional MR of the $\mathrm{ZnCoO}$ film $(-5 \%)$.

To realize electrical control of magnetotransport properties, we have applied AC and DC current simultaneously to the studied RMR devices. Fig. 3(a) shows the dependence of the detected voltage on the DC offset current measured under fixed $\mathrm{AC}=100 \mu \mathrm{A}$ and different magnetic fields. Different from the DC I-V curves in Fig. 2(a) where $V=0$ at $I=0$, the $\mathrm{I}-\mathrm{V}$ curves mixed with the AC component show $60.81 \mathrm{mV}$ rectification voltage at $\mathrm{I}=0$. Moreover, the rectification voltage at a fixed current depends on the magnetic field, indicating the obvious MR effect. The great modulation of magnetotransport properties of the RMR devices is further revealed in Fig. 3(b). The rectifying voltage represents the average outcome of the corresponding real time voltage over the time period of the applied alternating current, while under the application of a pure direct current, the detected conventional voltage drop always follow the current flow direction. Hence, the total detected rectification voltage

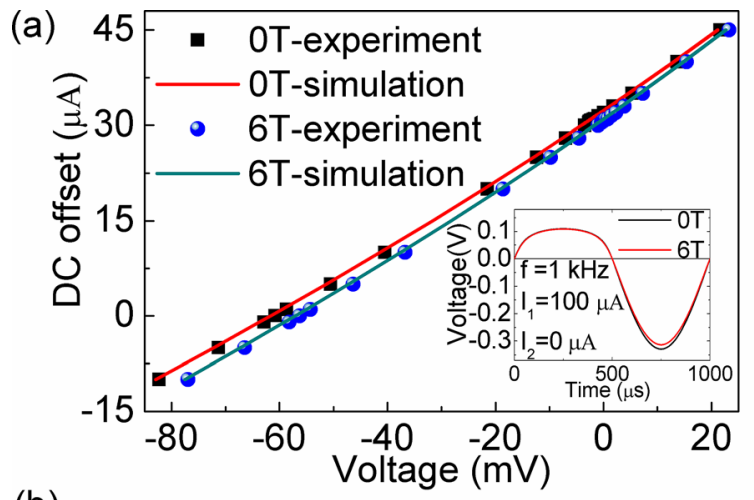

(b)
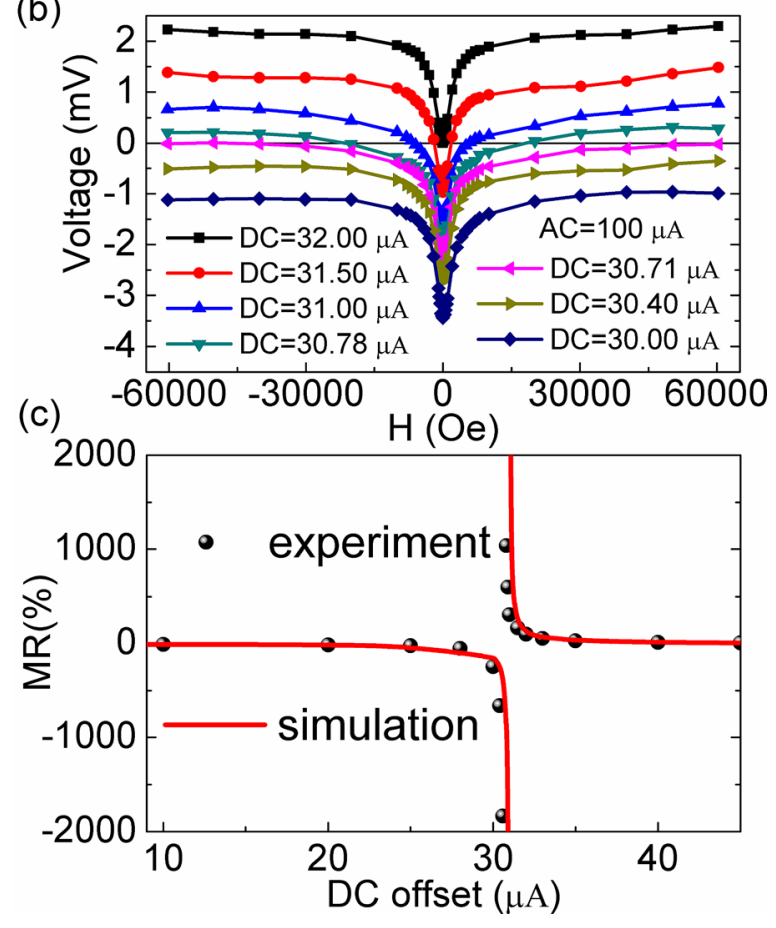

FIG. 3. (a) The experimental and theoretical simulated I-V curves mixed with the AC component. (b) The magnetic field dependent rectification voltage measured under fixed AC and variable DC offset. (c) The experimental and theoretical simulated DC offset dependence of the deduced MR from (a). Inset in (a) shows simulated time dependent voltage in one period under $\mathrm{AC}$ amplitude $=100 \mu \mathrm{A}, \mathrm{f}=1 \mathrm{kHz}$, and DC offset $=0 \mu \mathrm{A}$. can change from negative to positive with increasing DC offset though the shape of RMR curves remains nearly unchanged. By controlling the applied DC and AC, the $V_{H}$ could be tuned to near zero, which leads to the great enhancement of the detected magnetoresistance. As summarized in Fig. 3(c), not only extremely large RMR could be obtained but also the sign of the RMR could be reversed by changing the DC offset. The RMR ratio at a fixed AC of $100 \mu \mathrm{A}$ is $-11300 \%$ when $\mathrm{DC}$ offset $=30.71 \mu \mathrm{A}$, while it is $13500 \%$ when DC offset $=30.75 \mu \mathrm{A}$.

Fig. 4(a) shows the room temperature AC current amplitude dependence of the RMR at the fixed frequency of 1 $\mathrm{kHz}$. The RMR ratio decreases with the increasing AC amplitude and gradually tends to the conventional MR. It is clear that the RMR is larger than the conventional MR in a large current range from $10 \mu \mathrm{A}$ to $1 \mathrm{~mA}$. To investigate the frequency dependence of RMR, we built a half-wave rectifying circuit. As shown in the inset of Fig. 4(b), the load resistance consisted of a $1 \mathrm{M} \Omega$ resistor and $100 \mathrm{nF}$ capacitive load in parallel, which serve as both a low-pass filter and a DC load. A $50 \Omega$ resistor to ground was used at the input for transmission-line termination. We applied an $\mathrm{AC}$ voltage with a $1 \mathrm{~V}$ peak-to-peak value to the input terminal and measured the rectification voltage in the output terminal by varying the frequency. Fig. 4(b) shows the frequency dependence of the detected rectification voltage. For commercial diodes, the rectification voltage is constant at $0.2 \mathrm{~V}$ between $5 \mathrm{kHz}$ and $5 \mathrm{MHz}$. Meanwhile, the rectification voltage is constant at $0.1 \mathrm{~V}$ between $5 \mathrm{kHz}$ and $7 \mathrm{MHz}$ for the RMR device,
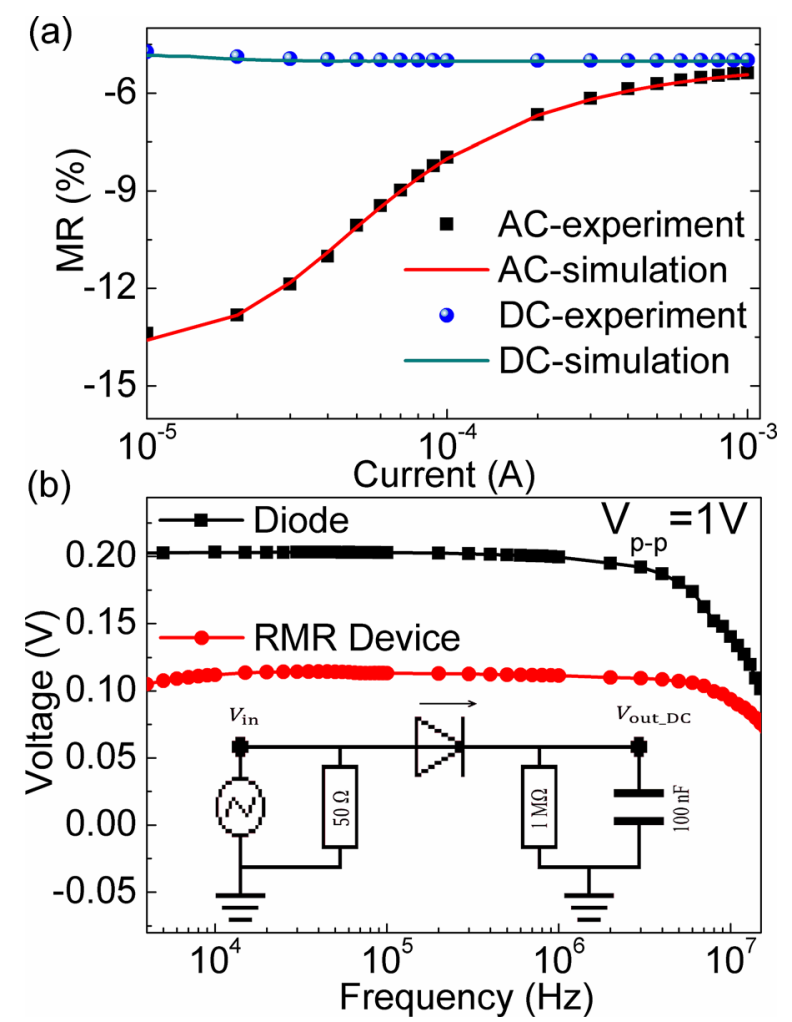

FIG. 4. (a) The experimental and theoretical simulated current amplitude dependence of the RMR (marked as AC). As a comparison, the experimental and theoretical simulated conventional MR (marked as DC) was shown. (b) The frequency dependent rectification voltage for the commercial diode and RMR device measured under $\mathrm{V}_{\mathrm{p}-\mathrm{p}}$ of $1 \mathrm{~V}$. The half-wave rectifier circuit setup is schematically shown in the inset in (b). 
revealing that the work frequency of the RMR device is high up to $7 \mathrm{MHz}$. Here, two points are worthy of mention. First of all, the diminution of rectification voltage in the RMR device is due to the decreasing asymmetry of device resistance between positive and negative applied current. Secondly, the cutoff frequency of the RMR device slightly increases up to $7 \mathrm{MHz}$ compared with that of the commercial diode. The cutoff frequency of the diode could be described by

$$
\mathrm{f}_{\mathrm{c}}=\frac{1}{2 \pi \mathrm{R}_{\mathrm{s}} \mathrm{C}_{\mathrm{J}}},
$$

where $R_{s}$ and $C_{J}$, respectively, represent the series resistance and junction capacitance. According to the I-V curve of the RMR device in Fig. 2(a), the parallel resistance of the RMR device is smaller than that of the commercial diode, which results in a slight increase of the cutoff frequency.

In order to clarify the mechanism of the RMR device and realize quantitative description, a theoretical model was established. As shown in Fig. 1(a), the linear I-V curves of the $\mathrm{ZnCoO}$ film obey Ohm's law and can be well fitted by the equation

$$
\mathrm{I}_{\mathrm{M}}(\mathrm{t})=\mathrm{G}(\mathrm{H}) \cdot \mathrm{V}(\mathrm{t}) .
$$

Here, $\mathrm{G}(\mathrm{H})$ varies with the applied magnetic field and describes the magnetoresistance of the $\mathrm{ZnCoO}$ film. The fitting parameters $\mathrm{G}(0 \mathrm{~T})=0.3005 \times 10^{-3} \Omega^{-1}$ and $\mathrm{G}(6 \mathrm{~T})=0.3156$ $\times 10^{-3} \Omega^{-1}$ represent the conductance under $0 \mathrm{~T}$ and $6 \mathrm{~T}$ magnetic fields, respectively. As shown in the inset of Fig. 1(c), the I-V curve of the commercial diode can be well fitted by the exponential equation

$$
\mathrm{I}_{\mathrm{D}}(\mathrm{t})=\mathrm{I}_{\mathrm{S}}\left(\mathrm{e}^{\alpha \mathrm{V}(\mathrm{t})}-1\right)
$$

which is similar to the Shockley equation in the ideal p-n junctions. Here, $\frac{1}{\alpha}=\mathrm{nV}_{\mathrm{T}}=2.6214 \times 10^{-2} \mathrm{~V}$, in which $\mathrm{V}_{\mathrm{T}}=\frac{\mathrm{k}_{\mathrm{B}} \mathrm{T}}{\mathrm{q}}=2.5852 \times 10^{-2} \mathrm{~V}$ is the thermal voltage at $300 \mathrm{~K}$ and $n \stackrel{\mathrm{q}}{=} 1.014$ is the ideality factor. $\mathrm{I}_{\mathrm{s}}=1.0628 \times 10^{-6} \mathrm{~A}$ represents the reverse saturation current. It is clear that Equation (3) can well describe the rectification effect of a diode without magnetoresistance.

Accordingly, the total current of the parallel circuit of the $\mathrm{ZnCoO}$ film and diode can be described by the equation

$$
\mathrm{I}(\mathrm{t})=\mathrm{I}_{\mathrm{M}}(\mathrm{t})+\mathrm{I}_{\mathrm{D}}(\mathrm{t})=\mathrm{I}_{\mathrm{s}}\left(\mathrm{e}^{\alpha \mathrm{V}(\mathrm{t})}-1\right)+\mathrm{G}(\mathrm{H}) \cdot \mathrm{V}(\mathrm{t}) .
$$

In principle, all the information about MR and rectification effect is included in Equation (4). In the meanwhile, the real time current $I(t)$ in our experiments was applied in the following form:

$$
\mathrm{I}(\mathrm{t})=\mathrm{I}_{1} \sin (2 \pi \mathrm{ft})+\mathrm{I}_{2} .
$$

In Equation (5), both the $\mathrm{AC}$ current $\mathrm{I}_{1}$ with frequency $\mathrm{f}$ and the DC offset $I_{2}$ are applied to the devices. Equation (5) could reduce to pure $\mathrm{AC}$ current if $\mathrm{I}_{1} \neq 0$ and $\mathrm{I}_{2}=0$, and pure DC current if $\mathrm{I}_{1}=0$ and $\mathrm{I}_{2} \neq 0$. By combining Equations (4) and (5), we could get the time dependent voltage $V(t)$ for any given current $I(t)$. As shown in the inset of Fig. 3(a), the $\mathrm{V}(\mathrm{t})$ curves under $0 \mathrm{~T}$ and $6 \mathrm{~T}$ were simulated by using the experimental parameters $\mathrm{I}_{1}=100 \mu \mathrm{A}, \mathrm{I}_{2}=0 \mu \mathrm{A}$, and $\mathrm{f}=1 \mathrm{kHz}$.

The rectification voltage represents the average outcome of the corresponding real time voltage $\mathrm{V}(\mathrm{t})$ over the time period $\mathrm{T}$ of the applied AC current, i.e.,

$$
\overline{\mathrm{V}}=\frac{1}{\mathrm{~T}} \int_{0}^{\mathrm{T}} \mathrm{V}(\mathrm{t}) \mathrm{dt} .
$$

In this case, the rectification magnetoresistance can be defined as

$$
\mathrm{RMR}=\frac{\overline{\mathrm{V}_{\mathrm{H}}}-\overline{\mathrm{V}_{0}}}{\overline{\mathrm{V}_{\mathrm{H}}}} \times 100 \% .
$$

Here, $\overline{\mathrm{V}_{\mathrm{H}}}$ and $\overline{\mathrm{V}_{0}}$ are the rectification voltage detected with and without applied magnetic field, respectively. Combining Equations (4)-(7), all the experimentally observed I-V curves, RMR, and the electrical manipulation of RMR can be well described, as shown in Figs. 2(a), 3(a), 3(c), and 4(a). One significant advantage of our engineered RMR device is that we can design the rectification magnetoresistance only using the physical parameters of the single diode and MR component. We could expect more amazing functions, such as realizing nonvolatile memory by using the tunneling magnetoresistance component and diode in parallel, which provide an additional degree of freedom to improve the device performance. However, some challenges need to be overcome before the realistic application of the RMR effect, such as large thermal effect and integration problems. If we apply an alternating current of $100 \mu \mathrm{A}$ to the studied devices, the maximum instantaneous power is on the order of $10 \mu \mathrm{W}$. By optimizing both the MR component and the commercial diode, it is possible to further reduce the total resistance of the devices and hence reduce the thermal effect, which invites further investigations and is beyond present study.

In summary, we not only demonstrate RMR by connecting a $\mathrm{ZnCoO}$ magnetoresistance film and a commercial diode in parallel but also obtain remarkable modulation of RMR from $-11300 \%$ to $13500 \%$ by simultaneously applying AC and DC to the device. Furthermore, a theoretical model was established without introducing any adjustable parameters, which can quantitatively describe the experimentally observed RMR and electrical modulation of RMR. This work may provide an alternative approach to exploit emergent multifunctions in existing MRAMs, magnetic sensors, and magnetic logic devices by introducing the RMR to modern integrated circuits technology.

We acknowledge the financial support from the National Basic Research Program of China No. 2013CB922303 and 2015CB921502, the key program of NSFC No. 11434006, 111 Project No. B13029, and the general program of NSFC No. 11374187.

${ }^{1}$ J. Grollier, V. Cros, A. Hamzic, J. M. George, H. Jaffrès, A. Fert, G. Faini, J. Ben Youssef, and H. Legall, Appl. Phys. Lett. 78, 3663 (2001).

${ }^{2}$ S. Mangin, D. Ravelosona, J. A. Katine, M. J. Carey, B. D. Terris, and E. E. Fullerton, Nat. Mater. 5, 210 (2006). 
${ }^{3}$ W. G. Wang, M. Li, S. Hageman, and C. L. Chien, Nat. Mater. 11, 64 (2012).

${ }^{4}$ S.-W. Jung, W. Kim, T.-D. Lee, K.-J. Lee, and H.-W. Lee, Appl. Phys. Lett. 92, 202508 (2008)

${ }^{5}$ S. S. P. Parkin, M. Hayashi, and L. Thomas, Science 320, 190 (2008).

${ }^{6}$ T. Zhao, A. Scholl, F. Zavaliche, K. Lee, M. Barry, A. Doran, M. P. Cruz,

Y. H. Chu, C. Ederer, N. A. Spaldin, R. R. Das, D. M. Kim, S. H. Baek, C.

B. Eom, and R. Ramesh, Nat. Mater. 5, 823 (2006).

${ }^{7}$ D. Lebeugle, D. Colson, A. Forget, M. Viret, A. M. Bataille, and A. Gukasov, Phys. Rev. Lett. 100, 227602 (2008).

${ }^{8}$ Y. H. Chu, L. W. Martin, M. B. Holcomb, M. Gajek, S. J. Han, Q. He, N. Balke, C. H. Yang, D. Lee, W. Hu, Q. Zhan, P. L. Yang, A. Fraile-Rodriguez, A. Scholl, S. X. Wang, and R. Ramesh, Nat. Mater. 7, 478 (2008).

${ }^{9}$ S. Polisetty, W. Echtenkamp, K. Jones, X. He, S. Sahoo, and C. Binek, Phys. Rev. B 82, 134419 (2010).

${ }^{10}$ H. G. Zhou, X. L. Fan, F. L. Wang, C. J. Jiang, J. W. Rao, X. B. Zhao, Y. S. Gui, C.-M. Hu, and D. S. Xue, Appl. Phys. Lett. 104, 102401 (2014).

${ }^{11}$ D. Chiba, F. Matsukura, and H. Ohno, Appl. Phys. Lett. 89, 162505 (2006).

${ }^{12}$ D. Chiba, M. Sawicki, Y. Nishitani, Y. Nakatani, F. Matsukura, and H. Ohno, Nature 455, 515 (2008).

${ }^{13}$ S. M. Wu, S. A. Cybart, P. Yu, M. D. Rossell, J. X. Zhang, R. Ramesh, and R. C. Dynes, Nat. Mater. 9, 756 (2010).

${ }^{14}$ Y. Yamada, K. Ueno, T. Fukumura, H. T. Yuan, H. Shimotani, Y. Iwasa, L. Gu, S. Tsukimoto, Y. Ikuhara, and M. Kawasaki, Science 332, 1065 (2011).
${ }^{15}$ K. Shimamura, D. Chiba, S. Ono, S. Fukami, N. Ishiwata, M. Kawaguchi, K. Kobayashi, and T. Ono, Appl. Phys. Lett. 100, 122402 (2012).

${ }^{16}$ Y. Y. Wang, X. Zhou, C. Song, Y. N. Yan, S. M. Zhou, G. Y. Wang, C. Chen, F. Zeng, and F. Pan, Adv. Mater. 27, 3196 (2015).

${ }^{17}$ G. Chen, C. Song, C. Chen, S. Gao, F. Zeng, and F. Pan, Adv. Mater. 24, 3515 (2012).

${ }^{18}$ C. Bi, Y. H. Liu, T. Newhouse-Illige, M. Xu, M. Rosales, J. W. Freeland, O. Mryasov, S. F. Zhang, S. G. E. te Velthuis, and W. G. Wang, Phys. Rev. Lett. 113, 267202 (2014).

${ }^{19}$ Y. Q. Xiong, W. P. Zhou, Q. Li, M. C. He, J. Du, Q. Q. Cao, D. H. Wang, and Y. W. Du, Appl. Phys. Lett. 105, 032410 (2014).

${ }^{20}$ K. Zhang, Y. L. Cao, Y. W. Fang, Q. Li, J. Zhang, C. G. Duan, S. S. Yan, Y. F. Tian, R. Huang, R. K. Zheng, S. S. Kang, Y. X. Chen, G. L. Liu, and L. M. Mei, Nanoscale 7, 6334 (2015).

${ }^{21}$ K. Zhang, H. H. Li, P. Grünberg, Q. Li, S. T. Ye, Y. F. Tian, S. S. Yan, Z. J. Lin, S. S. Kang, Y. X. Chen, G. L. Liu, and L. M. Mei, Sci. Rep. 5, 14249 (2015).

${ }^{22}$ D. Yang, F. Wang, Y. Ren, Y. Zuo, Y. Peng, S. Zhou, and D. Xue, Adv. Funct. Mater. 23, 2918 (2013).

${ }^{23}$ C. M. Xiong, Y. G. Zhao, B. T. Xie, P. L. Lang, and K. J. Jin, Appl. Phys. Lett. 88, 193507 (2006).

${ }^{24}$ C. d. Buttet, M. Hehn, F. Montaigne, C. Tiusan, G. Malinowski, A. Schuhl, E. Snoeck, and S. Zoll, Phys. Rev. B 73, 104439 (2006).

${ }^{25}$ S. S. Yan, J. P. Liu, L. M. Mei, Y. F. Tian, H. Q. Song, Y. X. Chen, and G. L. Liu, J. Phys.: Condens. Matter 18, 10469 (2006). 